\title{
The molecular mechanism of the type IVa pilus motor
}

\author{
Matthew McCallum ${ }^{1,2}$, Stephanie Tammam ${ }^{2}$, Ahmad Khan ${ }^{1}$, Lori L. Burrows ${ }^{3 *}$, P. \\ Lynne Howell ${ }^{1,2 *}$ \\ ${ }^{1}$ Department of Biochemistry, University of Toronto, Toronto, ON M5S 1A8, Canada \\ ${ }^{2}$ Program in Molecular Medicine, The Hospital for Sick Children, Toronto, ON M5G \\ 0A4, Canada. \\ ${ }^{3}$ Department of Biochemistry and Biomedical Sciences and the Michael G. DeGroote \\ Institute for Infectious Disease Research, McMaster University, Hamilton, ON L8N 3Z5, \\ Canada.
}

\begin{abstract}
Type IVa pili are protein filaments essential for virulence in many bacterial pathogens; they extend and retract from the surface of the cells to pull the bacteria forward with unprecedented force. They are used for attachment, swarming and twitching motility, biofilm formation, up-regulation of other virulence factors in response to surface sensing, and natural competence. The motor subcomplex assembles the pilus, and it is composed of the platform protein PilC and the ATPase PilB. Here we report the structure of the core ATPase domains of Geobacter metalloreducens PilB bound to ADP and the non-hydrolysable ATP analogue, AMP-PNP, at 3.4 and 2.3 $\AA$, respectively. These structures, determined under non-saturating nucleotide conditions, reveal important differences in nucleotide binding between chains. Analysis of these differences revealed the sequential turnover of nucleotide, and the corresponding domain movements. Our data indicate a clockwise rotation of the central sub-pores of PilB, which through interactions with PilC, would support the assembly of a right-handed helical pilus. Our analysis suggests a counterclockwise rotation of PilT's sub-pores that would enable righthanded pilus disassembly. This model provides insight into how this family of ATPases can power pilus extension and retraction with extraordinary forces.
\end{abstract}

